Inzwischen haben die chinesischen Kommunisten eingesehen, daß der große Fehler, der den Aufstand Hongkongs nicht wie in Macao zum Sieg führte, die mangelnde Mobilisation der Massen Hongkongs wạ. Streiks im Hafen und öffentlichen Verkehr sind gescheitert. Eine Intensivierung in dieser Richtung scheint die nächste wichtige Aufgabe der chinesischen Kommunisten in Hongkong zu sein. Daher ist eine kurze Ruhepause für Hongkong nicht ausgeschlossen. Hongkong bleibt zwar noch britische Kolonie, doch das Alltagsleben wird immer "roter" werden.

DR. C. L. YU

\title{
VERFASSUNG UND POLITISCHE ENTWICKLUNG IN BRASILIEN
}

Die neue brasilianische Verfassung vom 24. Januar 1967 trat am 15. März 1967 in Kraft, dem gleichen Tage, an dem auch der neue Staatspräsident sein Amt antrat. Sie ersetzt die Verfassung von 1946. Deren Struktur war im Verlauf der Revolution gegen den immer mehr unter linksradikalen Einfluß geratenen. Präsidenten Goulart im Jahre 1964 unter Berufung auf Revolutionsrecht ${ }^{1}$ bereits wesentlich geändert worden. Die neue Verfassung hielt die damals dem Staatspräsidenten eingeräumten Sonderbefugnisse nicht aufrecht; er kann nicht mehr politische Rechte entziehen und Mandate aberkennen.

Die Übergangsbestimmungen ${ }^{2}$ halten jedoch die verfassungsändernden Verordnungen sowie die auf ihnen fußenden einfachen Gesetze und Rechtshandlungen aufrecht und entziehen sie der richterlichen Nachprüfung. Insoweit behalten diese ihre Wirkung für die Zukunft. Nur die eigentlichen verfassungsändernden Bestimmungen, die sie enthielten, sind durch das das neue Grundgesetz abgelöst.

Umstritten ist dabei, wie die gewandelte Rechtslage den Umfang der vorher entzogenen politischen Rechte und die Sanktionen für Zuwiderhandlung beeinflußt. Heute hat der Entzug der politischen Rechte den Verlust des aktiven und passiven Wahlrechts sowie eines Mandats und Amtes zur Folge ${ }^{3}$. Die bisherigen Bestimmungen untersagten dagegen auch politische Betätigung und Äußerungen in der Offentlichkeit ${ }^{4}$; bei Verstößen konnte ein $Z$ wangswohnsitz angewiesen werden.

Es war nun die Frage, ob jemandem, dem auf Grund der früheren Bestimmungen die politischen Rechte entzogen waren, nach Inkrafttreten der neuen Verfassung die politische Betätigung verwehrt bleibt und ob die Zuweisung eines Zwangswohnsitzes weiterhin statthaft ist. In der Habeas-Corpus-Klage eines Journalisten entschied die Bundesjustiz zweiter Instanz (Tribunal Federal de Recursos) Anfang September 1967, daß seine Rechtslage sich nach den bisherigen Bestimmungen richtet, auch wenn die Zuwiderhandlung erst nach dem Inkrafttreten der neuen Verfassung erfolgt ist. Die Entscheidung erging mit knapper Mehrheit (6:5); einer der Richter führte aus, daß die gegenwärtige Verfassung nach Maßgabe ihres revolutionären Ursprungs $\mathrm{zu}$ interpretieren sei. Die politische Bedeutung des Urteils liegt in der Zurückweisung des Versuches, die von der revolutionären Regierung getroffenen Maßnahmen zu.entkräften.

\footnotetext{
1 Der vom Höchsten Revolutionskommando, den Ministern der drei Waffengattungen, am 9. 4. 1964 erlassene Erste Verfassungsakt betont in seiner Präambel: Die Revolution braucht sich nicht mittels des Kongresses zu legitimieren. Vielmehr erhält dieser seinerseits die gesetzliche Grundlage durch die verfassunggebende Macht, die allen Revolutionen innewohnt.

2 Artikel 173 der Verfassung.

3 Artikel $144 \S 1$ der Verfassung.

- Artikel 16 des Zweiten Revolutionären Verfassungsakts.
} 
Den ersten Entwurf der neuen Verfassung, der von einer aus vier hervorragenden Verfassungsrechtlern bestehenden Kommission ausgearbeitet worden war, verwarf die Regierung, weil er die Verwirklichung der revolutionären Ziele nicht hinreichend berücksichtige. Der zweite vom Justizminister verfaßte Entwurf wurde dem Parlament in außerordentlicher Sitzung vorgelegt. Die Regierung wünschte den Kongreß mitarbeiten zu lassen, um Vorwürfen zu begegnen, die Verfassung sei aufgezwungen. Andererseits gab sie dem Parlament nicht freie Hand, sondern bestand darauf, daß die von der Revolution geschaffene Machtverteilung, der Vorrang der Exekutive in einem repräsentativ-demokratischen System, erhalten bliebe. Die für die Beratung zur Verfügung stehende Zeit wurde befristet.

Die Eile erklärt solche Widersprüche im Text wie den zwischen Artikel $31 § 2$ und Artikel $79 \int 2$. Nach dem ersten treten Senat und Abgeordnetenkammer bei gemeinsamen Tagungen unter Leitung des Senatspräsidiums zusammen, während der zweite dem Vizepräsidenten der Republik den Vorsitz gewährt. Der Konflikt wurde durch die Persönlichkeiten, die diese Ämter innehatten, verschärft. Er mußte politisch gelöst werden, ehe er seine rechtliche Bereinigung finden konnte. Die Bestimmungen wurden dahingehend interpretiert, daß der Vizepräsident der Republik bei gemeinsamen Sitzungen beider Häuser von Amts wegen am Senatspräsidium teilnimmt und den Vorsitz führt, während der Senatspräsident nur die Sitzungen des Senats selbst leitet.

Die Schwächung des Parlaments hat unmittelbare Folgen auch für die Stellung der einzelnen Mitglieder. Ihr Einfluß ist gesunken. Ein Sitz im Kongreß gibt nicht mehr dieselbe Machtbasis im heimatlichen Wahlbezirk wie früher, und die Entlegenheit der neuen Hauptstadt Brasília vergrößert diese Benachteiligung. Die Gouverneure der Gliedstaaten beherrschen die Parteiorganisationen in weit stärkerem Maße, und sie können die Aussichten der Wiederwahl erheblich beeinflussen, auch wenn sie selbst für die folgende Periode nicht wählbar sind. $\mathrm{Da}$ das politische Leben Brasiliens nicht so sehr von den Weltanschauungen der Parteien wie von den Persönlichkeiten der Politiker geprägt wird, kommt es diesen darauf an, sich ihre Gefolgschaft zu erhalten.

Das wird durch die im Oktober 1965 erfolgte Auflösung der Parteien ${ }^{5}$ erschwert. Damals gab es dreizehn, von denen einige noch verschiedene Flügel hatten. An ihre Stelle traten zwei Blocks, die $\mathrm{ARENA}^{6}$ auf Seiten der Regierung und $\mathrm{MDB}^{7}$ als Opposition. Beide haben sich inzwischen als Parteien konstituiert. Die Bildung weiterer Parteien ist durch Mindesterfordernisse an Stimmzahlen und Mandaten in beiden Häusern erschwert ${ }^{8}$. Bei der gegenwärtigen Konstellation fehlen für die Schaffung einer dritten Partei die politischen Voraussetzungen, da sie kaum erwarten könnte, am Machtprozeß entscheidend teilzunehmen. Bezeichnend ist, daß ursprünglich sogar Schwierigkeiten bestanden, genügend Mitglieder für den Oppositionsblock zu finden.

Das Zweiparteiensystem erzwingt eine Polarisation in Regierungsblock und Opposition und verlangt den Ausgleich der Gegensätze innerhalb der beiden Großparteien. Es steht dem Bedürfnis der Politiker nach eigener Hausmacht im Wege. So wird erwogen, wieder „sublegendas“, d. h. Flügel mit eigener Organisation im Rahmen der Großparteien zuzulassen, die es bei den Wahlen 1966 bereits gegeben

\footnotetext{
5 Artikel 18 des Zweiten Revolutionären Verfassungsakts.

- Aliança Renovadora Nacional - Allianz zur Erneuerung des Staates.

7 Movimento Democrático Brasileiro - Brasilianische Demokratische Bewegung.

8 Artikel 149 VII der Verfassung; vgl. $\$ 6$ (4) des deutschen Bundeswahlgesetzes.

- Artikel 9 des Vierten Zusätzlicien Verfassungsakts.
} 
hatte ${ }^{9}$. Koalitionen sind verboten ${ }^{10}$. Die „sublegendas“ sind als Verbindung mehrerer Listen derselben Partei anzusehen, die bei der Sitzverteilung als eine gelten $^{11}$.

Auch heute noch werden die Politiker nach ihrer Zugehörigkeit zu den früheren Parteien eingeordnet. Die Wiedereinführung von „sublegendas“ könnte zu erneuter Gruppenbildung nach Art der aufgelösten Parteien führen. Deshalb neigt die Regierung dazu, die „sublegendas" nur während der Wahlzeit, nicht aber als Parlamentsfraktionen zuzulassen. Die endgültige Regelung wird von der Stärke der Kräfte abhängen, die das Wahlrecht und damit den politischen Prozeß gestalten ${ }^{12}$. Ausgeschlossen vom Machtprozeß sind diejenigen, denen die politischen Rechte entzogen sind, aber auch Männer wie der Journalist und Politiker Carlos Lacerda, der als damaliger Gouverneur des Staates Guanabara (Stadt Rio de Janeiro) wesentlich zur Revoultion von 1964 beigetragen hat, dann aber nicht bereit war, sich einem der beiden Blocks einzuordnen. Es ist natürlich, daß diese Kräfte nach der Teilnahme am Machtprozeß streben. Da ihnen die Betätigung innerhalb des Systems verwehrt ist, suchen sie nach Wegen, die nicht zum Zusammenstoß mit der Regierung führen, aber Möglichkeiten neben der bestehenden Staatsorganisation eröffnen. So entstand die "Breite Front" (frente ampla), die eine Opposition neben der Opposition darstellt. Sie versucht, Elemente beider Großparteien an sich zu ziehen. Sie ist keine dritte Partei und kann nicht erwarten, es zu werden. Dazu müßten sich Senatoren und Abgeordnete in genügender Anzahl ihr anschließen und ihren bisherigen Einfluß aufgeben.

Gestützt wird die Breite Front yon den beiden früheren Staatspräsidenten Kubitscheck und Goulart, denen die politischen Rechte entzogen sind, sowie Lacerda, der seine Hoffnungen auf die Präsidentschaft - wenn überhaupt - nur in direkten Wahlen verwirklichen kann. Wie schon das Revolutionsrecht schreibt auch die neue Verfassung die indirekte Wahl vor ${ }^{13}$. Diese Aufgabe erhöht zwar das Ansehen des Kongresses, entspricht aber nicht der Tradition. Weiten Kreisen gilt nur die direkte Wahl als demokratisch. Damit hat die Opposition einen Kampfruf, und die „Erklärung von Montevideo"14, die von Lacerda und Goulart unterzeichnet wurde, verlangt die "Wiederaufnahme des demokratischen Prozesses durch direkte Wahl“. Kubitschek und Goulart können sich nur bei einer Amnestie, die in naher Zukunft nicht zu erwarten ist, wieder politisch betätigen. Ihre Unterschrift unter das Bündnis mit Lacerda kommt zunächst diesem zugute, der erwarten darf, Teile der Anhängerschaft seiner Partner für sich zu gewinnen. Da sich jedoch andere Führer der ehemaligen Arbeiterpartei (PTB) dem Bündnis mit dem Manne widersetzen, der $1964 \mathrm{zu}$ dem Sturze Goularts beigetragen hat, erscheint es fraglich, ob die Unterschrift Goularts Lacerda viel nützen wird. Sie kann ihm auch insofern schaden, als in anderen Kreisen das Bündnis als Zeichen des Opportunismus gewertet wird. Jedenfalls erreichen die Beteiligten, daß die Wählerschaft sie nicht vergißt, auch wenn die Breite Front keinen unmittelbaren Einfluß auf die Gestaltung des Machtprozesses ausüben kann.

Die Breite Front könnte jedoch die Regierung gegen deren Wunsch zu einer Verhärtung des politischen Kurses veranlassen. Damit würde sie gerade das verhindern, was sie anstrebt: die Festigung des demokratischen Prozesses. Die doppeldeutigen

\footnotetext{
10 Artikel 149 VIII der Verfassung.

$11 \mathrm{Vgl}$. $\delta 7$ (2) des deutschen Bundeswahlgesetzes.

12 Dazu Loewenstein, Karl: Verfassungslehre, Tübingen 1959, S. 275.

1s Artikel 76 der Verfassung. Das Wahlkollegium besteht aus den Mitgliedern des Kongresses sowie

Delegierten der Landtage nach Maßgabe der Größe der jeweiligen Wählerschaft in den Gliedstaaten.

14 Vom 25. September 1967.
} 
Formulierungen der „Erklärung von Montevideo“ lassen andererseits befürchten, daß eine solche Entwicklung unausgesprochenes Ziel sein könnte. Denn wie Celso Furtado ${ }^{15}$ bereits 1962 schrieb, kann die Einführung einer Diktatur von rechts, die die politische Struktur verhärtet, die Bedingungen für eine wirksame revolutionäre Machtgruppierungen von marxistisch-leninistischem Typ schaffen. Die Verfassung von 1967. bietet die Möglichkeit, im Zusammenspiel zwischen starker Präsidentschaft und verantwortungsbewußtem Kongreß einer solchen Entwicklung vorzubeugen.

DR. H. Jolowicz 